\title{
Frequency of Polymorphisms of Genes Coding for HIV-1 Co-Receptors CCR5 and CCR2 in a Brazilian Population
}

Patrícia Munerato, Maria Lúcia Azevedo, Maria Cecília Araripe Sucupira, Regina Pardini, Gedson Humberto Novaes Pinto, Márcia Catroxo, Inara Espinelli Souza and Ricardo Sobhie Diaz

\author{
Retrovirology Laboratory, Federal University of São \\ Paulo, UNIFESP, Biomedical Science Institute, \\ University of São Paulo,USP, São Paulo, SP, Brazil
}

\begin{abstract}
Entry of human immunodeficiency type 1 virus (HIV-1) into target cells requires both $\mathrm{CD}_{4}$ and one of the chemokine receptors. Viruses predominantly use one, or occasionally both, of the major co-receptors CCR5 and CXCR4, although other receptors, including CCR2B and CCR3, function as minor co-receptors. A 32-nucleotide deletion $(\Delta 32)$ within the $\beta$-chemokine receptor 5 gene (CCR5) has been described in subjects who remain uninfected despite extensive exposition to HIV-1. The heterozygous genotype delays disease progression. This allele is common among Caucasians, but has not been found in people of African or Asian ancestry. A more common transition involving a valine to isoleucine switch in transmembrane domain I of CCR2B (64I), with unknown functional consequences, was found to delay disease progression but not to reduce infection risk. As the Brazilian population consists of a mixture of several ethnic groups, we decided to examine the genotype frequency of these polymorphisms in this country. There were $11.5 \%$ CCR5 heterozygotes among the HIV-1 infected population and $12.5 \%$ among uninfected individuals, similar to data from North America and Western Europe. The prevalence of CCR2$64 I$ homozygotes and heterozygotes was 0.06 and $15.2 \%$, respectively, also similar to what is known for North America and Western Europe.

Key Words: CCR5, CCR2B, HIV-1 infection, polymorphisms.
\end{abstract}

Attachment of HIV-1 to cells is mediated between specific sequences of the gp120 envelope glycoprotein and the $\mathrm{CD}_{4}$ receptor, which normally is a receptor for the major histocompatibility complex class II (MHC-II) molecules. Although the $\mathrm{CD}_{4}$ is the best-known receptor, it alone is not sufficient for HIV-1 entry into cells. Evidence suggesting additional receptors first came from the fact that HIV-1 could not efficiently replicate in other animal cells engineered to express human $\mathrm{CD}_{4}[1]$. The discovery of co-receptors for HIV-1 entry, such as CXCR4,CCR5, CCR2, and CCR3, which are chemokine receptors, have provided insights into this phenomenon [2].

Received on 20 March 2003; revised 28 July 2003.

Address for correspondence: Dr. Ricardo Sobhie Diaz. Federal University of São Paulo. Rua Pedro de Toledo, 781, 16 andar, Vila Clementino, São Paulo/SP, Brazil. Zip code: 04039-032.

The Brazilian Journal of Infectious Diseases 2003;7(4):236-240 (c) 2003 by The Brazilian Journal of Infectious Diseases and Contexto Publishing. All rights reserved.
Physiologically, chemokine receptors mediate the chemotaxis of T-cells and phagocytes to areas of inflammation [3]. Upon ligand binding, the receptors transduce an intracellular signal that results in the rapid mobilization of intracellular calcion. Each of the eight known chemokine receptors is a $\mathrm{G}$ protein with a characteristic pattern of ligand binding $[3,4]$. The receptor CCR5, which also serves as a major coreceptor for macrophage-tropic HIV-1 virus, binds the $\beta$-chemokines RANTES (regulated on activation, normal $\mathrm{T}$ expressed and secreted), MIP- $1 \alpha$ (macrophage inflammatory protein) and MIP-1 $\beta$ [5]. The ligands for CCR2, which also serves as a minor co-receptor for HIV-1 entry, are MCP-1, 2, 3, 4, and 5 (monocyte chemoattractant protein) [6].

In fact, different HIV-1 strains have distinct cell tropisms; viruses with T-cell tropism utilize the CXCR4 co-receptor, while those with selective macrophagemonocyte tropism utilize the CCR5 or CCR2 co- 
receptors $[2,7]$. The strain usually involved in the primary infection - macrophage-tropic isolates - is the strain that uses the CCR5 or CCR2 co-receptors expressed on mucosal dendritic cells. The more cytopathic (SI) T-cell-tropic strains that evolve during the course of infection, and that precipitate accelerated T-cell depletion and progression to AIDS, utilize the CXCR4 receptor. "Dual tropic" HIV-1 strains appear during the course of infection; such strains may be intermediates between macrophage- and T-cell-tropic populations. This transition in cell tropism, involving viral adaptation, which enables broader co-receptor use, may be a key step in progression to AIDS [7].

Another insight that came from the discovery of co-receptors is the observation that a few individuals remain unaffected by HIV-1 despite multiple highrisk sexual exposures to the virus [8-10]. It has been shown that the $\mathrm{CD}_{4}^{+} \mathrm{T}$-cells of these individuals are highly resistant in vitro to the entry of primary macrophage-tropic virus, but are readily infected with transformed T-cell-line-adapted viruses [11]. Subsequent to this discovery, a 32-base pair deletion $(\triangle 32)$ in the CCR5 gene was identified in some Caucasians [12,13]. This mutated allele causes truncation of the CCR5 co-receptor on lymphoid cell surfaces. In the homozygous state, this truncated allele is epidemiologically related to the resistance of some highly exposed individuals to HIV-1 infection. An association of delayed disease progression with CCR5 $\Delta 32$ has also been reported [14]. The frequency of the allele CCR 532 has been estimated in various ethnic groups [14]. Among Caucasians, approximately $15 \%$ are heterozygotes and $1 \%$ are homozygotes. In contrast, the deleted allele is rare among persons of African, Asian and Hispanic origin [14].

The polymorphisms in the CCR2 gene involve a point mutation that results in a switch from valine to isoleucine at transmembrane protein position 64 , described as CCR264I. One major study [14] found a correlation between CCR2B 64I polymorphisms and disease progression, although these findings were not confirmed by a second study [15]. Nonetheless, CCR2B 64I polymorphisms have no protective effect against HIV-1 infection [14,15].
Brazil is considered an ethnically diverse country, with individuals of Caucasian, African, Asian and native American origin, and interracial marriages are common. We decided to analyse CCR5 and CCR2B polymorphisms in this population.

We made a cross-sectional molecular epidemiology study to determine the prevalence of individuals homozygous and heterozygous for the mutation coding for the 32-pair deletion in the CCR5 chemokine receptor gene (CCR5 $\Delta 32$ ), and for a valine to isoleucine switch in transmembrane domain I of CCR2B (CCR2 64I).

\section{Materials and Methods}

Clinical samples. One hundred and eighty-three HIV1-positive samples were obtained from outpatient clinics at the Federal University of São Paulo in São Paulo, Brazil. We also analyzed 115 samples from women with unknown HIV-1 status, as a control group. These samples were taken in accordance with the ethics standards of the Ethics Committee of the Federal University of São Paulo and with the Helsinki Declaration of 1975, revised in 1983.

DNA purification. DNA was extracted from frozen whole blood using a QIAamp Blood Kit (QIAGEN Inc, Santa Clarita, CA), using the methodology indicated by the manufacturer.

CCR5 genotyping. A portion of the CCR5 gene was amplified by PCR from genomic DNA and analyzed on a 5\% Acrylamide gel, yielding a $189 \mathrm{bp}$ fragment for the wild type allele, and a $157 \mathrm{bp}$ fragment for the deleted allele (Figure 1A). PCR primers flanking the 32-nucleotide deletion were 5' CAA AAA GAA GGTCTTCAT TAC ACC 3', and 5' CCT GTG CCT CTT CTT CTC ATT TCG 3', as described previously [5]. The PCR reaction mixture the final $100 \mu \mathrm{L}$ contained $50 \mathrm{mM} \mathrm{MgCl}_{2}, 10$ $\mathrm{mM}$ of dNTPs, 20 pmol of each primer, and 0.5 unit of Taq Polymerase in $1 \mathrm{X}$ reaction buffer (Gibco BRL, Gaithersburg MD). Each PCR amplification consisted of 40 cycles, with five cycles of $94^{\circ} \mathrm{C}$ for $1 \mathrm{~min}, 55^{\circ} \mathrm{C}$ for 1 min, and $72^{\circ} \mathrm{C}$ for $1.5 \mathrm{~min}$, followed by 35 cycles of $94^{\circ} \mathrm{C}$ for $30 \mathrm{~s}, 60^{\circ} \mathrm{C}$ for $30 \mathrm{~s}$, and $72^{\circ} \mathrm{C}$ for $45 \mathrm{~s}$. 
CCR2 genotyping. Amplification of a portion of the CCR 2 gene by PCR was performed with primers 5' TTG TGG GCA ACA TGa TGG, which has a cytosine substituted by an adenine (in lower case) and 5' GAG CCC ACA ATG GGA GAG TA, as described previously [14]. The final 128-bp product was analyzed for Restriction Fragment Length Polymorphisms (RFLP) using Bsa BI enzyme, which generated 110- and $18 \mathrm{bp}$ fragments when an isoleucine was present instead of a valine at position 64 in CCR2. These products were genotyped in a $5 \%$ acrylamide gel (Figure 1B).

\section{Results}

To determine the frequency of polymorphism of the CCR5 gene, 183 HIV-1-infected individuals were tested at the Medical School of São Paulo. One hundred percent of the samples were positive by PCR. Consistent with previous findings, the analysis in the $5 \%$ acrylamide gel showed that none of the infected subjects were homozygous for the $\Delta 32$ allele $(\Delta / \Delta)$. The frequency of heterozygotes $(\Delta /+)$ in this population was $11.5 \%$, while $88.5 \%$ were wild type $(+/+)$ for the deletion (21 and 162 individuals, respectively). The control population was also investigated to compare these data with possibly uninfected individuals. These samples were obtained from a population at risk for HIV-1 infection; 115 women were included in this study. The results were similar to those found in Caucasian populations in North America and western Europe: homozygous $-0 \%$, heterozygous $-12.5 \%$ and wild type $-87.5 \%$ (Table 1 )

The same sample of infected individuals was investigated for CCR2 gene polymorphism. PCR positivity was $97.27 \%$. Analysis of the control group was deemed unnecessary since the prevalence of CCR2 $64 I$ seems to be the same in infected and uninfected individuals. One individual among the 178 enrolled in this study was homozygous $(64 I / 64 I)$ for the $64 I$ genotype $-0.56 \%, 27$ were heterozygous $(64 I /+)-15.17 \%$, and 150 were wild type $(+/+)-$ $84.26 \%$ (Table 1).

\section{Discussion}

Although anecdotical cases of HIV-positive CCR5 532 homozygous individuals have been described [16,17], numerous epidemiological studies have confirmed that the absence of full-length CCR5 confers protection to HIV infection. Recent studies of transfusion recipients and hemophiliacs indicate

Table 1. Genotypic frequency of the CCR5 and CCR2 polymorphisms in a HIV-1 infected (CCR5 and CCR2) and a control population (CCR5). The wild type CCR5 is represented by $(+)$, whereas the 32 base pair deletion is represented bt $\Delta$. The wild type CCR 2 is represented by $(+)$, and the valine to isoleucine substitution at position 64 is represented by $64 I$.

\begin{tabular}{lcccc}
\hline & \multicolumn{4}{c}{ CCR5 Genotype } \\
\cline { 2 - 5 } & $\Delta / \Delta$ & $\Delta /+(\%)$ & $+/+(\%)$ & Total (\%) \\
\hline Control (HIV-1 status unknown) & 0 & $15(13.0)$ & $100(87.0)$ & $115(100 \%)$ \\
Infected (HIV-1 seropositive) & 0 & $21(11.5)$ & $162(88.5)$ & $183(100 \%)$ \\
\hline & \multicolumn{4}{c}{ CCR2 Genotype } \\
\cline { 2 - 5 } & $\mathbf{6 4 I / 6 4 I ( \% )}$ & $\mathbf{6 4 I / + ( \% )}$ & $+/+(\%)$ & Total $(\boldsymbol{\%})$ \\
Infected (HIV-1 seropositive) & $1(0.6)$ & $27(15.17)$ & $150(88.5)$ & $178(100 \%)$ \\
\hline
\end{tabular}


Figure 1. Analyses of CCR5 and CCR2B amplification.

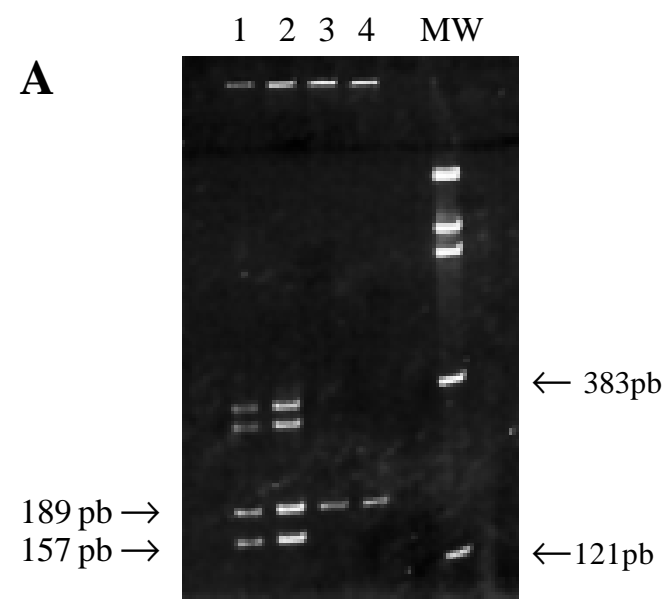

A. CCR5 was amplified by PCR from genomic DNA of outpatient clinics and in the control group. Primers that flank the deletion were used to generate wild-type and deleted fragments of $189 \mathrm{bp}$ and $157 \mathrm{bp}$, respectively. The position of the wild-type $(3,4)$ and heterozygous deleted fragments $(1,2)$ is indicated by arrows in the 5\% acrylamide gel. Sizes of marker fragments (MW) are indicated to the right in base pairs.

that this resistance occurs when the parenteral route is involved [18].

More recently, a second mutation in CCR5 that has a prevalence of $1 \%$ in the European population was described. A single-base-pair mutation, m303, introduces a stop codon that prevents cell surface expression of a functional CCR5 receptor. Thus, $\mathrm{m} 303$ homozygosity, or its association with the CCR5 $\Delta 32$ allele, could account for some of the unexplained cases of HIV-1 resistance in individuals who carry an apparent wild type CCR5 or who are heterozygous [21]. Polymorphism of other genes coding for other co-receptors related to MT strain infection, such as CCR2, apparently have no influence on the incidence of HIV-1 infection.

There is also a relationship between genetic polymorphism in host cell co-receptors and disease progression. The onset of AIDS seems to be postponed in individuals heterozygous for the CCR5

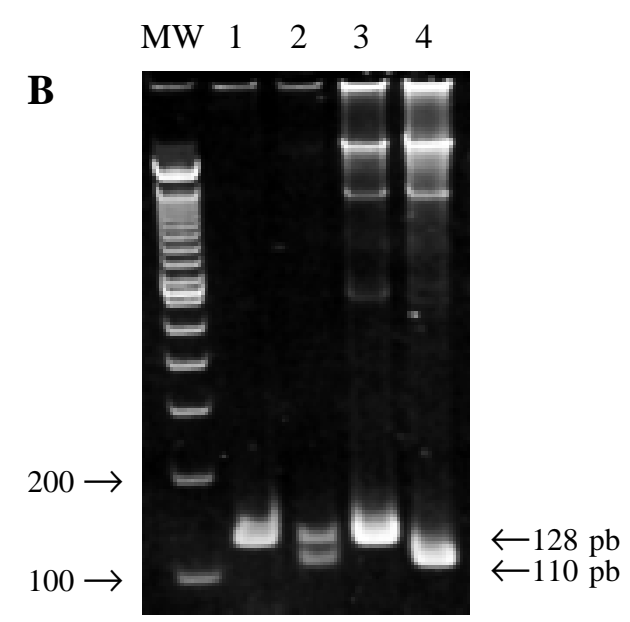

B. Genomic DNA from outpatient clinics and the control group was amplified by PCR to generate $128 \mathrm{bp}$ fragments. The final product was digested by Bsa BI enzyme, which generated 110 and $18 \mathrm{bp}$ fragments when an isoleucine was present instead of a valine at position 64 in CCR2B. These products were separated in the $5 \%$ acrylamide gel. This gel shows wild-type fragments in wells 1 and 3, and fragments heterozygous and homozygous for the genotype change in wells 2 and 4, respectively. Sizes of marker fragments (MW) are indicated to the left in base pairs.

$\Delta 32$ allele $[14,19,20]$. However, identification of the heterozygous condition for CCR5 accounts for only a small proportion of the long-term non-progressors that remain AIDS-free for 10 to 20 years after HIV-1 infection. As an example, more than $60 \%$ of long-term non-progressors are homozygous for the common allele CCR5+/+ [13]. The other chemokine receptor gene, CCR2, also has some degree of polymorphism that might be related to disease progression. A point mutation, CCR2 64I, occurs at an allele frequency of 10 to $15 \%$ among Caucasians and African Americans. Data from one large cohort indicate that HIV-1 infected individuals carrying this allele progressed to AIDS two to four years later than individuals homozygous for the common allele [14], whereas another study failed to detect this association [18].

This is the first description of the prevalence of CCR5 and CCR2 polymorphisms in a Brazilian population. The Caucasian pattern of CCR5 allele 
polymorphism prevails in this population, even though is a great deal of ethnic mixture in this country.

\section{Acknowledgments}

This work was supported by PRONEX (41.96.0943), and FAPESP (98/07719-9). We thank Cecilia Cheng-Mayer for providing the CCR2 prototype samples.

\section{References}

1. Simon J.H., Somoza C., Schockmel G.A., et al. Rat CD4 mutant containing the gp120-binding site mediates human immunodeficiency virus type 1 infection. J. Exp. Med 1993; 177:949-54.

2. Clapham P.R., Blanc D., Weiss R.A. Specific cell surface requirements for the infection of CD4-positive cells by human immunodeficiency virus types 1 and 2 and by Simian immunodeficiency virus. Virology 1991;181:703-15.

3. Horuk R. Molecular properties of the chemokine receptor family. Trends Pharmacol Sci 1994;15:159-65.

4. Schall T.J. Biology of the RANTES/SIS cytokine family. Cytokine 1991;3:165-8.

5. Samson M., Labbe O., Mollereau C., et al. Molecular cloning and functional expression of a new human $\mathrm{C}$ C-chemokine receptor gene. Biochemistry 1996;35:3362-7.

6. D'Souza M.P., Harden V.A. Chemokines and HIV-1 second receptors. Confluence of two fields generates optimism in AIDS research. Nat Med 1996;2:1293-300.

7. Koot M., Van't Wout A.B., Kootstra N.A., et al. Relation between changes in cellular load, evolution of viral phenotype, and the clonal composition of virus populations in the course of human immunodeficiency virus type 1 infection. J Infect Dis 1996;173:349-54 .

8. Paxton W., Martin S.R., Tse D., et al. Relative resistance to HIV-1 infection of CD4 lymphocytes from persons who remain uninfected despite multiple high risk sexual exposure. Nat Med 1996;2:412-7.

9. Dean M., Carrington M., Winkler C., et al. Genetic restriction of HIV-1 infection and progression to AIDS by a deletion allele of the CKR5 structural gene. Science 1996;27:273:1856-62.

10. Liu R., Paxton W.A., Choe S., et al. Homozygous defect in HIV-1 coreceptor accounts for resistance of some multiply-exposed individuals. Revista 1996.

11. Rowland-Jones S., Sutton J., Ariyoshi K., et al. HIV-specific cytotoxic T-cells in HIV-exposed but uninfected Gambian women. Nat Med 1995;1:50-64.
12. Wilkinson D.A., Operskalski E.A., Busch M.P., et al. A 32-bp deletion within the CCR5 locus protects against transmission of parenterally acquired human immunodeficiency virus but does not affect progression to AIDS-defining illness. J Infect Dis 1998; 178:1163-6.

13. Zimmerman P.A., Buckler-White A., Alkhatib G., et al. Inherited resistance to $\mathrm{HIV}-1$ conferred by an inactivating mutation in $\mathrm{CC}$ chemokine receptor 5; Studies in populations with contrasting clinical phenotypes, defined racial background, and quantified risk. Mol. Med 1997;3:23-36.

14. Smith M.W., Dean M., Carrington M., et al. Contrasting genetic influence of CCR 2 and CCR5 variants on HIV-1 infection and disease progression. Science 1997; 15;277(5328):959-65

15. Michael N.L., Louie L.G., Rohrbaugh A.L., et al. The role of CCR5 and CCR2 polymorphisms in HIV-1 transmission and disease progression. Nat Med 1997; $3: 1160-4$

16. O'Brien T.R., Winkler C., Dean M., et al. HIV-1 infection in a man homozygous for CCR-5 delta 32. [letter] Lancet 1997;349:1219.

17. Theodorou I., Meyer L., Magierowska M., et al. HIV-1 infection in an individual homozygous for CCR-5 delta 32. Seroco Study Group [letter]. Lancet 1997;349:1219-20.

18. Wilkinson D.A., Operskalski E.A., Busch M.P.., et al. A 32-bp deletion within the CCR5 locus protects against transmission of parenterally acquired human immunodeficiency virus but does not affect progression to AIDS-defining illness. J Infect Dis 1998;178:1163-6.

19. Huang Y., Paxton W.A., Wolinsky S.M., et al. The role of mutant CCR-5 allele in HIV-1 transmission and disease progression. Nat Med 1996; 2: 1240-43.

20. Ioannidis J.P., Rosenberg P.S., Goedert J.J., et al. International Meta-Analysis of HIV Host Genetics. Effects of CCR5-Delta32, CCR2-64I, and SDF-1 3'A alleles on HIV-1 disease progression: An international meta-analysis of individual-patient data. Ann Intern Med 2001; 135:782-95.

21. Quillent C., Oberlin E., Braun J., et al. HIV-1-resistance phenotype conferred by combination of two separate inherited mutations of CCR5 gene. Lancet 1998;351:14-8. 